\title{
Centralized and Decentralized Balance Group Optimization in Electricity Markets with Demand Response
}

\author{
Evangelos Vrettos*, Frauke Oldewurtel*, Matteo Vasirani ${ }^{\dagger}$ and Göran Andersson* \\ ${ }^{*}$ Power Systems Laboratory, ETH Zurich, Zurich, Switzerland \\ Email: $\{$ vrettos $\mid$ oldewurtel|andersson $\} @$ eeh.ee.ethz.ch \\ ${ }^{\dagger}$ Distributed Information Systems Laboratory, EPFL, Lausanne, Switzerland \\ Email: matteo.vasirani@epfl.ch
}

\begin{abstract}
In this paper, the potential of using Demand Response (DR) to minimize balancing energy costs of Balance Groups (BGs) in electricity markets is investigated. Two algorithms are developed based on direct and price-based control concepts, respectively, to control an aggregated pool of office buildings. The direct control algorithm is set up as a centralized Model Predictive Control (MPC) problem yielding an optimal control sequence. This is used as a benchmark for a decentralized price control scheme, which is suboptimal, but still provides a good performance with much lower communication requirements compared to the benchmark. The two approaches are compared using a case study and conclusions regarding their advantages and disadvantages are drawn based on simulation results. The results show that with proper exploitation of the flexibility of office building aggregations significant balancing cost reductions can be achieved with only limited communication which is, in particular, respecting privacy requirements.
\end{abstract}

\section{INTRODUCTION}

\section{A. Background}

The assumed setting is a liberalized electricity market in which generation units and consumers are organized in $\mathrm{Bal}$ ance Groups (BGs). ${ }^{1}$ BGs are virtual aggregations of market players for accounting and billing purposes only, i.e., loads and generators are not necessarily concentrated in the same region. Several BGs are subsumed in a control area which is supervised by a Transmission System Operator (TSO). BGs can conduct energy transactions among them or with BGs of other control areas. The TSO is responsible for keeping system frequency at $50 \mathrm{~Hz}$ and maintain the agreed crossborder power schedules with neighboring TSOs.

In this market setting, each BG in the control area submits a day-ahead power exchange schedule to the TSO, i.e., a profile of the BG net position during the next day with a time resolution of 15 minutes. Due to load or generation prediction errors and unexpected events, some BGs may deviate from the submitted schedules during real-time operation. This will create a generation-load mismatch and eventually frequency deviation from $50 \mathrm{~Hz}$. The TSO compensates for frequency

\footnotetext{
${ }^{1}$ The BG concept is used in Switzerland but also in other European countries, although it might be given a different name. An example is Germany where the name Balancing Responsible Party (BRP) is used instead.
}

deviations by employing power reserves from contracted regulating units. The cost for employing these reserves is passed to the particular BGs that are responsible for the deviations. Thus, in principle, each $\mathrm{BG}$ has an economic incentive to follow its submitted power exchange schedule as closely as possible.

A widespread use of smart metering infrastructure is expected to enable nearly real-time power measurements in various grid locations. With this information, a particular BG can track its schedule in order to minimize balancing energy costs with either of the three following possibilities: (a) adjusting the setpoints of dispatchable power plants, (b) trading in intra-day markets, and (c) using Demand Response (DR) [1]. This paper focuses on (c) and proposes one centralized and one decentralized algorithm to enable BG optimization by exploiting the flexibility of large office buildings.

\section{B. Related Work}

The potential of using direct and price-based load control schemes for DR has been the subject of several publications. Reference [2] presents an overview of recent research in this field. Some papers, such as [3], [4], focus on optimal building operation in a time-varying electricity price environment. Other works, such as those proposed in [5]-[7] investigate concurrent optimization of end-consumers' electricity bill and producers' generation cost. It is shown that there exist timevarying prices that achieve a reasonable tradeoff between building-level optimality and social optimality. A lot of work has been reported on system level applications of price-based DR including peak shaving and load shifting, e.g., [8]-[13]. In [14], [15] the authors investigate coordination schemes for consumers that jointly offer demand reduction services that guarantee monetary profits for the participants, while [16] considers the formation of coalitions of energy consumers with near-complementary consumption restrictions that act in the market as a single virtual energy consumer.

\section{Contribution}

We address two aspects which have not been treated in the aforementioned papers: (a) explicit minimization of balancing energy costs using DR and (b) quantitative comparison of 
direct and price-based control algorithms. In particular, we formulate centralized and decentralized algorithms that use the thermal inertial of office buildings as a virtual storage to mitigate the schedule deviations of a $\mathrm{BG}$. The idea is to reduce buildings' aggregate consumption when the BG power exchange is lower than the scheduled one (i.e., BG is short) and increase it when the $\mathrm{BG}$ power exchange is higher than the scheduled one (i.e., BG is long). This can be an attractive approach for three main reasons. First, the energy consumption of office buildings can be adapted by controlling their Heating, Ventilation, and Air Conditioning (HVAC) system. Due to the inherent thermal storage capacity, the load can in principle be shifted in time without compromising the occupant comfort [11], [12]. Second, the problem is solved in a local, distributed manner without relying on complex market operations. And third, the need for expensive power reserves from conventional generators is minimized.

We first formulate the problem as a deterministic centralized Model Predictive Control (MPC) problem assuming full state feedback at every time step. Solving this problem provides the optimal solution for the assumed performance index and can be used as a benchmark. Then, we propose a decentralized price-based control scheme for the same problem, which requires less frequent measurements from the buildings and hence reduces concerns about privacy and communication limits, but shows an acceptable performance compared to the benchmark.

\section{PRoblem DESCRIPTION}

\section{A. Overview}

A BG is assumed to consist of generators (GEN), fluctuating Renewable Energy Resources (RES), non-controllable consumers (CSM) and a controllable aggregation of office buildings (BLD). A scheme of the considered BG is shown in Figure 1. The BG is managed by a Balance Group Manager (BGM) and the BLD is controlled by an aggregator through direct communication links. We further assume that the aggregator is the electric energy provider for BLD.

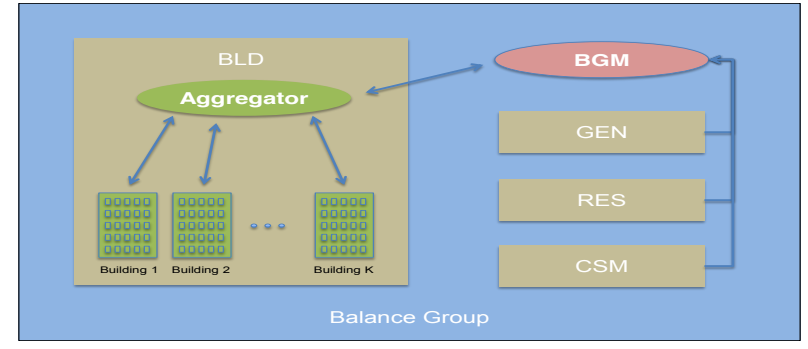

Fig. 1. A BG consisting of GEN, RES, CSM, and BLD; the latter being managed by an aggregator. The arrows indicate communication links.

\section{B. Modeling and Control of Buildings}

As building model we use a linear version of the model in [17], [18]. This model is linear since the cooling tower is not considered and the blind position is fixed. The system states are temperatures in the room, floor, and ceiling, the control inputs are heating power, cooling power, and lighting and the disturbances include weather conditions and internal heat gains due to people and equipment.

We assume that each building uses an MPC controller for determining its HVAC control inputs since thermal models are expected to be available for office buildings in the future. The objective of the controller is to minimize electricity costs by incorporating predictions about future disturbances, which are assumed known in this work. Thus, using a sufficiently long prediction horizon the MPC controller gives the optimal building response. Using MPC for building control is particularly well-suited for DR, since prices and/or varied constraints can be easily incorporated in the respective optimization problems.

\section{Control Approaches}

First, the ideal case with a central controller at the aggregator side that has two-way communication channels with all buildings and receives full state feedback at every time step (e.g. every 15 minutes) is considered in Section III. However, in reality, it might not be desirable to communicate frequently all state information for privacy reasons or due to communication constraints. Therefore, we propose a control scheme that uses price signals, which are broadcasted by the aggregator as incentives for the buildings to jointly minimize deviations from the schedule, and only requires measurements from buildings once per day. This is detailed in Section IV.

\section{Centralized Control}

In this approach, the central controller receives measurements from the power system as well as full state feedback (temperatures) and predictions for weather conditions and occupancy from all buildings in BLD. Let us denote by $P_{t}^{\mathrm{GEN}}$, $P_{t}^{\mathrm{RES}}, P_{t}^{\mathrm{CSM}}$ and $P_{t}^{\mathrm{BLD}}$ the GEN power, the RES power, the CSM load and the BLD load, respectively, at time step $t$. The scheduled BG power exchange for time step $t$ is denoted by $P_{t}^{\mathrm{sch}}$, whereas the actual BG net position is given by:

$$
P_{t}^{\mathrm{np}}=\underbrace{P_{t}^{\mathrm{GEN}}+P_{t}^{\mathrm{RES}}-P_{t}^{\mathrm{CSM}}}_{P_{t}^{\text {meas }}}-P_{t}^{\mathrm{BLD}} .
$$

Equation (1) implies that $P_{t}^{\mathrm{GEN}}, P_{t}^{\mathrm{RES}}$ and $P_{t}^{\mathrm{CSM}}$ are measured by the BGM at each time step $t$ using the smart metering infrastructure and that the measurements are communicated to the aggregator controller.

Let us denote the HVAC control inputs of building $b$ at time step $t$ by $u_{t}^{\mathrm{b}} \in \mathbb{R}^{n_{u}}$, its state by $x_{t}^{\mathrm{b}} \in \mathbb{R}^{n_{x}}$ and the disturbances by $\mathrm{v}_{t}^{\mathrm{b}} \in \mathbb{R}^{n_{\mathrm{v}}}$. The aggregate power consumption of all buildings is given by:

$$
P_{t}^{\mathrm{BLD}}:=\sum_{\mathrm{b}=1}^{L}\left(\xi^{\mathrm{b}}\right)^{T} u_{t}^{\mathrm{b}},
$$

where $L$ is the number of buildings in BLD and $\xi^{\mathrm{b}} \in \mathbb{R}^{n_{u}}$ is a scaling vector in order to take into account the different efficiencies of building actuators. With this notation, the BG schedule deviation can be expressed as:

$$
\Delta P_{t}=P_{t}^{\mathrm{sch}}-P_{t}^{\mathrm{np}}=P_{t}^{\mathrm{sch}}-P_{t}^{\mathrm{meas}}+P_{t}^{\mathrm{BLD}} .
$$




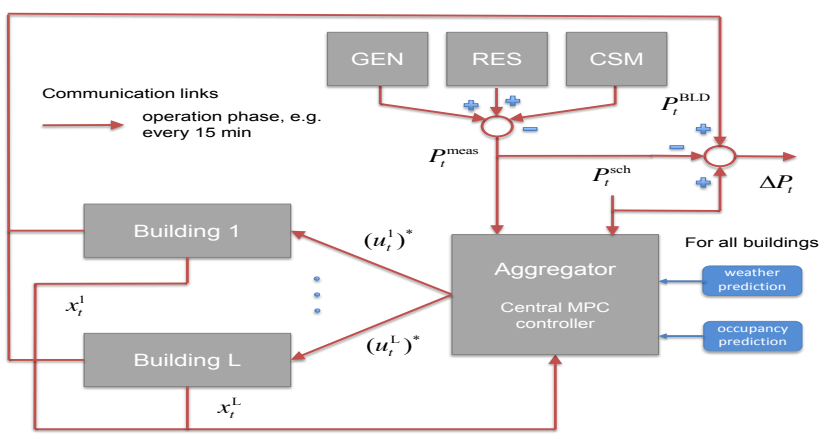

Fig. 2. Central MPC controller block diagram.

We assume that $P_{t}^{\text {meas }}$ stays constant throughout the time interval $[t, t+1)$, hence the goal is to obtain a suitable $P_{t}^{\mathrm{BLD}}$ to minimize the controller cost function. The cost function has to reflect two goals: (a) minimization of balancing energy costs (i.e., deviations from the scheduled power exchange, $\Delta P_{t}$ ) and (b) minimization of the total BLD electricity consumption. Since there are known costs for both of these components, one can directly formulate this in the optimization problem.

In the following, vectors and matrices that contain all predictions of a variable along the prediction horizon $N$ are denoted with bold letters, i.e. $\mathbf{x}_{t}^{\mathrm{b}}, \mathbf{u}_{t}^{\mathrm{b}}$ and $\mathbf{v}_{t}^{\mathrm{b}}$. By adopting this notation and combining (2) and (3) the centralized MPC problem looks as follows:

$$
\begin{array}{rr}
\min _{\mathbf{u}_{t}^{\mathrm{b}}} r_{t}\left|P_{t}^{\mathrm{sch}}-P_{t}^{\text {meas }}+\sum_{b=1}^{L}\left(\xi^{\mathrm{b}}\right)^{T} u_{t}^{\mathrm{b}}\right|+\sum_{b=1}^{L} c\left(\boldsymbol{\xi}^{\mathrm{b}}\right)^{T} \mathbf{u}_{t}^{\mathrm{b}} \\
\text { s.t. } \\
\mathbf{S}^{\mathrm{b}} \mathbf{u}_{t}^{\mathrm{b}} \leq \mathbf{s}^{\mathrm{b}} \quad \forall b \in 1 \ldots L \\
\mathbf{G}^{\mathrm{b}} \mathbf{x}_{t}^{\mathrm{b}} \leq \mathbf{g}^{\mathrm{b}} \quad \forall b \in 1 \ldots L \\
\mathbf{x}_{t}^{\mathrm{b}}=\mathbf{A} x_{0}^{\mathrm{b}}+\mathbf{B} \mathbf{u}_{t}^{\mathrm{b}}+\mathbf{E} \mathbf{v}_{t}^{b} \quad \forall b \in 1 \ldots L,
\end{array}
$$

where $|\cdot|$ denotes the absolute value operator, $r_{t}$ is the cost for balancing energy at time step $t, c \in \mathbb{R}$ is the electricity price, and matrices $\mathbf{A}, \mathbf{B}, \mathbf{E}, \mathbf{S}^{\mathrm{b}}, \mathbf{G}^{\mathrm{b}}$ and vectors $\mathbf{s}^{\mathrm{b}}, \mathbf{g}^{\mathrm{b}}$ are of appropriate sizes. Equations (5), (6) correspond to input and state constraints, whereas (7) describes the building dynamics.

The solution of this MPC problem is the optimal HVAC control input sequence for each building $\left(\mathbf{u}_{t}^{\mathrm{b}}\right)^{*}$. Only the first step of the sequence is applied to the buildings and then the procedure is repeated at the next time instant. The block diagram of the central MPC controller is shown in Figure 2.

\section{DeCEntralized CONTROL}

The aim of this algorithm is to provide an incentive for buildings to jointly mitigate the schedule deviations, without excessive communication requirements, by dynamically modifying the electricity price. It is assumed that all $L$ buildings have energy supply contracts with the aggregator at a specified electricity price $c$ and that the thermal models of all buildings are known to the aggregator. The transmitted price signal at time $t$ is denoted by $c_{t}^{\mathrm{tr}}$ and defined as:

$$
c_{t}^{\mathrm{tr}}:=c+\Delta c_{t} .
$$

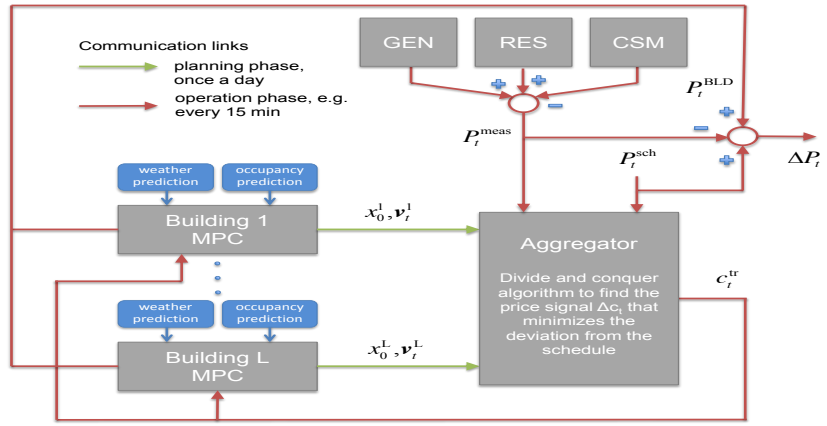

Fig. 3. Overview of the decentralized price-based control scheme.

Therefore, the goal of the aggregator is to determine a suitable $\Delta c_{t}$ for each time step $t$ so that the balancing energy costs are minimized. The buildings have a monetary incentive to reduce (increase) their electricity consumption in case $\Delta c_{t}>(<) 0$, i.e. the transmitted electricity price $c_{t}^{\text {tr }}$ becomes higher (lower) than the contracted price $c$.

The algorithm consists of two parts: the day-ahead planning phase at building level and the operation phase at aggregator side. Note that state feedback is not required at every time step and thus the communication burden is minimal. An overview of the control scheme is given in Figure 3.

The planning phase takes place at the end of each day. Each building $b$ sends its current state $x_{0}^{\mathrm{b}}$ as well as weather and occupancy predictions for the next day to the aggregator. In the operation phase all computations are done by the aggregator, which receives only $P_{t}^{\text {meas }}$ and $P_{t}^{\text {sch }}$ as inputs. Determining the optimal price signal, $\Delta c_{t}^{*}$, is actually the master problem of a bilevel optimization with the slave problem being the determination of the buildings' HVAC control inputs.

Due to the structure of the problem, that is the monotonicity of building response to price signals, the problem can be approximated by assuming that the aggregator can select $\Delta c_{t}$ from an ordered list of candidate price control signals, i.e., $\Delta c_{t} \in L_{\mathrm{c}}:=$ $[-c,-c+m,-c+2 m, \ldots, 0, m, 2 m, \ldots c-2 m, c-m, c]$, where $m \in \mathbb{Q}^{*}$ is a granularity factor ${ }^{2}$. Now, $\Delta c_{t}^{*}$ can be computed by applying a dichotomic divide and conquer algorithm (also known as binary search [19]) in the ordered list. The basic idea is that the set of possible price signals is sequentially bisected leading to a fast convergence to $\Delta c_{t}^{*}$ for the simplified problem, which is expected to be slightly suboptimal for the original problem. Note that the algorithm's complexity is $O\left(\log _{2} n\right)$ instead of $O(n)$, which is the complexity of a brute force search, where $n=2 \frac{c}{m}+1$ is the number of candidate price signals. In the simulations the value $m=0.5$ was chosen, which is a reasonable tradeoff between computational burden and solution accuracy.

It is assumed so far that the aggregator possesses the thermal models of the buildings participating in BLD. If the models are not known, demand-price elasticities can be precomputed by

\footnotetext{
${ }^{2}$ It is assumed that the lowest price is 0 and the highest $2 c$. The granularity factor $m$ is a design parameter providing tradeoff between solution accuracy and computational burden.
} 


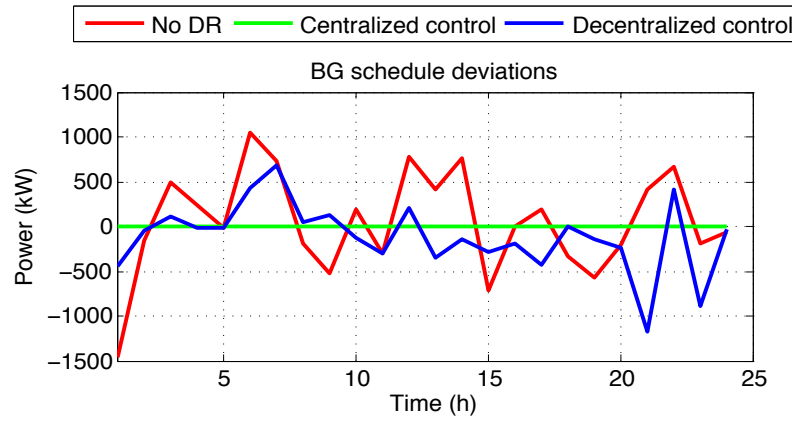

Fig. 4. BG schedule deviations without control, with centralized MPC and with decentralized price-based control.

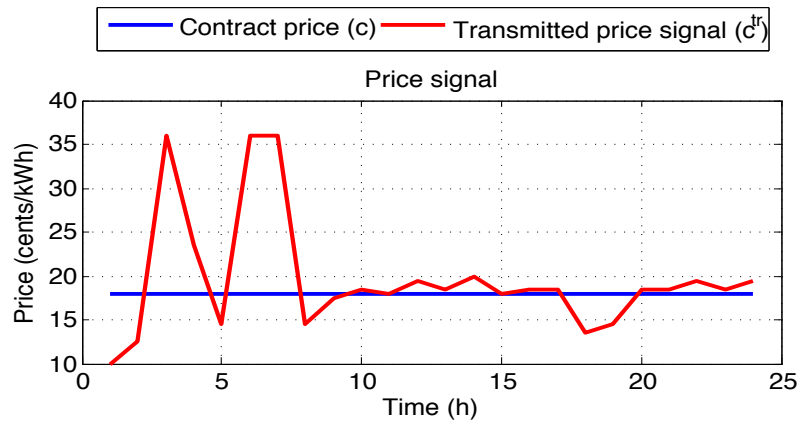

Fig. 5. The transmitted price signal in the decentralized control approach.

each building at the planning phase and communicated to the aggregator once per day to provide him with some information about buildings' responses to price signals. The elasticities are determined by the predicted demand changes, with respect to the baseline, in the presence of price changes $\Delta c_{t} \in L_{\mathrm{c}}$ for different time instances during the day.

\section{CASE Study}

Due to lack of actual BG data, a fictitious BG benchmark has been constructed to compare the developed control approaches. The CSM load and RES production are derived by taking the aggregated demand and wind power for year 2011 from Tennet ${ }^{3}$ and scaling them to reasonable magnitudes. The standard deviations of CSM and RES day-ahead prediction errors are assumed to be $3 \%$ and $15 \%$, respectively, which are typical values according to [20], [21]. The BG includes conventional generation with constant production corresponding to a base-load power plant, e.g., a nuclear power plant.

BLD consists of 32 office buildings of various HVAC systems, building standards, construction types, windows area fractions and internal gain magnitudes, as shown in Table I. For more details on the building types or the considered occupancy profiles the reader is referred to [18]. Note that the buildings are modeled as building zones in [18], whereas reasonable floor areas in the range 10000-30000 $\mathrm{m}^{2}$ are assumed in this work to represent office buildings. The weather predictions are assumed to be perfect, i.e., realizations equal the predictions. The corresponding weather data for Zurich

\footnotetext{
${ }^{3}$ Tennet is one of the four TSOs in Germany. The load and wind power data are publicly available under http://www.tennettso.de.
}

TABLE I

BUILDING TYPES

\begin{tabular}{|c||c|c|}
\hline Parameter & Option 1 & Option 2 \\
\hline HVAC system & radiator, no ventilation & $\mathrm{TABS}^{4}$, ventilation \\
Building standard & swiss average & passive building \\
Construction type & heavy & light \\
Window area fraction & high & low \\
Internal gains & high & low \\
\hline
\end{tabular}

for the year 2007 is obtained from MeteoSwiss [22]. The electricity market is considered by using the EPEX spot prices for Switzerland for 2012 [23] as well as the Swiss balancing energy pricing scheme [24]. All buildings in BLD are assumed to have electricity supply contracts with the aggregator at a price of $c=18$ cents $/ \mathrm{kWh}$.

\section{Simulation Results}

In this section, simulation results from the operation of the benchmark BG for a typical day in winter are presented to compare the developed algorithms. For the decentralized control approach, the simulations have been carried out assuming that the aggregator possesses the buildings' thermal models. The analysis is divided into three parts: (a) control performance, (b) economic investigation, and (c) assessment of buildings' flexibility. A discussion of the results of this work is then given in Section VII.

\section{A. Control Performance}

Figure 4 presents the $\mathrm{BG}$ schedule deviations for a day without DR, with centralized control and with decentralized control. The transmitted price signal for the decentralized approach is shown in Figure 5. The centralized MPC brings schedule deviations to zero for all hours during the considered day. The decentralized approach generally decreases the magnitude of deviations, however, the potential improvement is clearly lower compared to the centralized one. Also, note that the aggressive price signals during the first hours of the day lead to poorer control performance at the end of the day. Actually, there are instances when the decentralized control results in larger deviations compared to the case without DR.

\section{B. Economic Investigation}

The hourly balancing energy costs without DR, with centralized control and with decentralized control are shown in Figure 6. Table II presents a comparison of the BLD total electricity consumption, electricity costs and balancing costs among the three cases. Note that the electricity costs are computed using $c$ for the centralized control and $c_{t}^{\text {tr }}$ for the decentralized one.

The case without DR is energy optimal, that is the buildings consume the least amount of energy to stay within the comfort zone during this particular day. Despite a small increase in consumption, which is due to energy being shifted to the next day as well as numerical reasons, the consumption stays virtually constant in both control approaches. The electricity cost is practically the same in the case without DR and with

\footnotetext{
${ }^{4}$ TABS: Thermally Activated Building System for heating and cooling.
} 


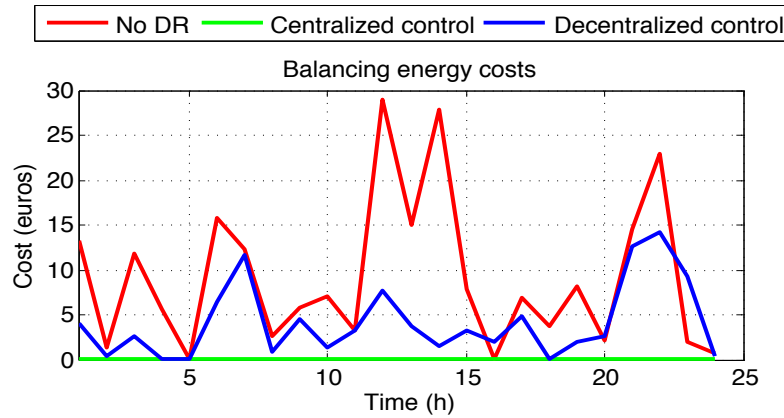

Fig. 6. Hourly balancing energy costs without control, with centralized MPC and with decentralized price-based control.

TABLE II

CONSUMPTION, ELECTRICITY COSTS AND BALANCING Costs

\begin{tabular}{|c||c|c|c|}
\hline & $\begin{array}{c}\text { Consumption } \\
\text { (MWh) }\end{array}$ & $\begin{array}{c}\text { Electricity } \\
\text { cost }(€)\end{array}$ & $\begin{array}{c}\text { Balancing } \\
\text { cost (€) }\end{array}$ \\
\hline No DR & 251 & 45185 & 220.08 \\
Centr. control & 250 & 44970 & 0 \\
Decentr. control & 247 & 45977 & 99.81 \\
\hline
\end{tabular}

the centralized control, whereas decentralized control results in slightly higher electricity cost. Balancing costs are reduced by $100 \%$ with the centralized approach and $55 \%$ with the decentralized one.

\section{Building Flexibility}

Figure 7 shows how the aggregate BLD load is shifted to mitigate BG schedule deviations with respect to the baseline consumption, i.e., the case without DR. During night hours the BLD load is low, therefore load changes even up to $90 \%$ are required to stay close to the schedule. On the contrary, during working hours load changes less than $5 \%$ are usually enough to compensate CSM and RES prediction errors.

The contributions of individual buildings to the hourly shifted load in the centralized and decentralized control approaches are shown in Figure 8. Buildings 9-16 and 25-32, which are passive (i.e., well insulated) practically do not react to control signals in any of the control approaches. In Figure 8(a) two typical building reactions are observed. Building 24 reduces its consumption from hour 3 to hour 6 , when the price is high, and compensates for the lost energy by increasing its consumption from hour 9 to hour 11 , when the price is lower. On the contrary, building 19 continuously decreases its consumption from hour 12 to hour 19 without compensating for the lost energy, which implies that its flexibility allows the energy to be shifted to the next day. From Figure 8(b) it can be seen that buildings with radiators (1-16) do not react to price control signals, whereas Swiss average buildings with TABS (17-24) are practically the only ones which can shift load in the decentralized approach.

\section{DISCUSSION}

\section{A. Control Performance}

The centralized approach exhibits an excellent potential for mitigation of $\mathrm{BG}$ schedule deviations but requires frequent state update from the buildings to the central controller. This

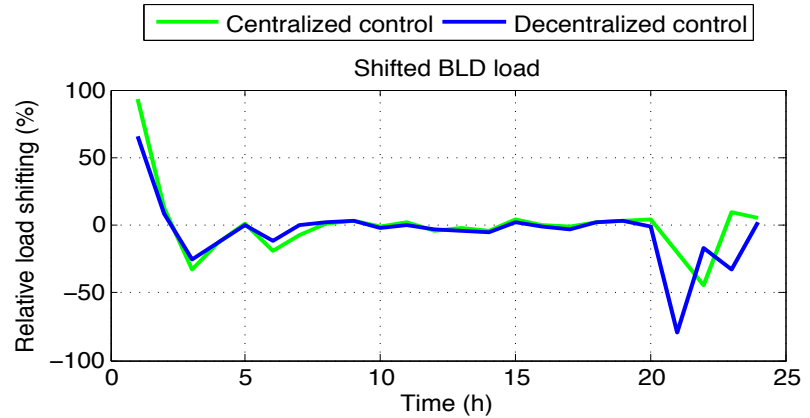

Fig. 7. BLD load shifting with respect to baseline consumption, i.e., the case without DR.

means that measurements or estimates of the temperatures in each room, and even in the walls, need to be communicated frequently to the aggregator. Apart from the obvious communication burden, this approach arises also privacy issues since the room occupancy can be reconstructed using the temperature measurements. An advantage of the centralized control is that it allows a direct tradeoff between electricity consumption and balancing costs and can be used in practice by aggregators to evaluate the flexibility of a given portfolio of controllable office buildings. For instance, our simulations show that the flexibility offered by BLD is sufficient for this particular BG.

On the other hand, the decentralized approach exhibits a lower, but still satisfying, potential for minimization of BG schedule deviations. It utilizes the buildings' models, however, no temperature measurements are needed during the day respecting privacy and reducing communication requirements.

It was observed that schedule deviations can even increase for particular hours with the decentralized control. The reason is the well-known herding effect, that is controllable buildings respond similarly to price signals, therefore it is likely that many of them reach simultaneously the boundaries of their comfort zones. In these cases, the aggregator might not be able to effectively control schedule deviations. This is mitigated in two ways in this paper: (a) an heterogenous BLD comprising buildings of different types is chosen, as shown in Table I, and (b) $\Delta c_{t}$ is determined by applying an optimization routine on a list of candidate price signals using a relatively high granularity factor $m$.

\section{B. Economic Investigation}

The economic analysis revealed that the centralized control minimizes balancing costs without increasing the BLD electricity cost. However, this is not the case for the decentralized control which increases the BLD electricity cost in order to reduce balancing costs. The reason is that the BG is short for most of the day and thus the average $c_{t}^{\mathrm{tr}}$ is greater than the contract price. This is not a surprising result since it has been previously reported in [11] that cost increases are possible if real-time pricing is used as an incentive mechanism for peak shaving. In practice, undesirable cost increases for buildings could be avoided by using a scaled version of the price signal time series with an average value less or equal to the contract price $c$ for the actual billing of the buildings. 

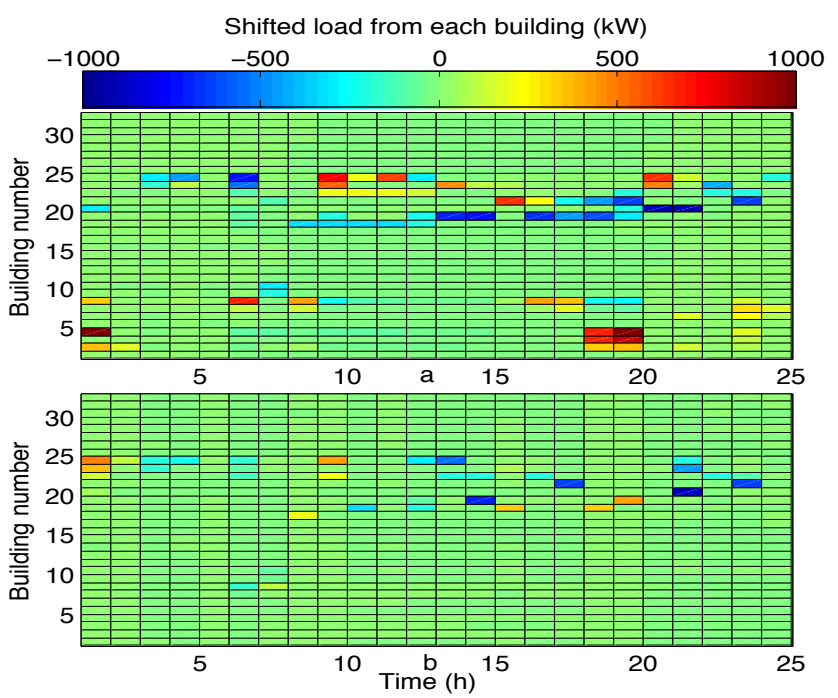

Fig. 8. Buildings' contributions to the total BLD hourly shifted load in centralized control (a) and decentralized control (b).

\section{Building Flexibility}

Figure 8(a) shows the optimal load shifting for the assumed balancing and electricity cost data and can be used to assess the flexibility of different types of buildings. First, passive buildings exhibit little potential for load shifting since internal gains are enough to compensate ambient losses and additional energy from heating actuators is not needed. Second, buildings with TABS (17-32) offer a greater flexibility compared to buildings with radiators (1-16), in particular when consumption must be reduced. This difference is more prolonged in Figure 8(b), where load shifting occurs practically only in buildings with TABS. The large flexibility in this case is the combined effect of slow TABS dynamics and high concrete thermal inertia, which allows efficient rescheduling of heating in the presence of a price signal.

\section{CONCLUSION}

In this paper, two control schemes based on demand response with aggregations of large office buildings are developed for balancing energy cost reduction in electricity markets. The centralized control demonstrates a performance benchmark, but requires a large communication burden and arises privacy issues. The decentralized price-based control provides acceptable performance at significantly reduced communication requirements and by respecting privacy. The simulation results show that there exists a large potential for balancing cost reduction with both control approaches. Future work will consider the uncertainties in RES and CSM predictions by formulating stochastic optimization problems to improve control performance.

\section{REFERENCES}

[1] S. Koch, M. Zima, and G. Andersson. Potentials and applications of coordinated groups of thermal household appliances for power system control purposes. In Proceedings of the IEEE-PES/IAS Conference on Sustainable Alternative Energy, Valencia, Spain, September 2009.

[2] D.S. Callaway and I.A. Hiskens. Achieving controllability of electric loads. Proceedings of the IEEE, 99(1):184-199, January 2011.
[3] A. J. Conejo, J. M. Morales, and L. Baringo. Real-time demand response model. IEEE Transactions on Smart Grid, 1(3):236-242, Dec. 2010.

[4] Y. Guo, M. Pan, and Y. Fang. Optimal power management of residential customers in the smart grid. IEEE Transactions on Parallel and Distributed Systems, 23(9):1593-1606, 2012.

[5] T. Cui, H. Goudarzi, S. Hatami, S. Nazarian, and M. Pedram. Concurrent optimization of consumer's electrical energy bill and producer's power generation cost under a dynamic pricing model. In Conference on Innovative Smart Grid Technologies (ISGT 2011), pages 1-6, District of Columbia, USA, January 2012.

[6] N. Li, L. Chen, and S.H. Low. Optimal demand response based on utility maximization in power networks. In Proceedings of the IEEE PES General Meeting, pages 1-8, Detroit, Michigan, USA, July 2011.

[7] S. Datchanamoorthy, S. Kumar, Y. Ozturk, and G. Lee. Optimal time-of-use pricing for residential load control. In IEEE International Conference on Smart Grid Communications (SmartGridComm 2011), pages 375-380, Brussels, Belgium, October 2011.

[8] A. Molderink, V. Bakker, M. G. C. Bosman, J. L. Hurink, and G. J. M. Smit. Management and control of domestic smart grid technology. IEEE Transactions on Smart Grid, 1(2):109-119, September 2010.

[9] A. Molderink, V. Bakker, M. G. C. Bosman, J. L. Hurink, and G. J. M. Smit. On the effects of mpc on a domestic energy efficiency optimization methodology. In Proceedings of the IEEE International Energy Conference, pages 120-125, Manama, Bahrain, December 2010.

[10] D. L. Ha, F. F. de Lamotte, and Q. H. Huynh. Real-time dynamic multilevel optimization for demand-side load management. In Proceedings of the IEEE International Conference on Industrial Enginnering and Engineering Management, pages 945-949, Singapore, December 2007.

[11] F. Oldewurtel, A. Ulbig, A. Parisio, G. Andersson, and M. Morari. Reducing peak electricity demand in building climate control using realtime pricing and model predictive control. In Proceedings of the IEEE Conference on Decision and Control, Atlanta, Georgia, USA, Dec. 2010.

[12] F. Oldewurtel, A. Ulbig, M. Morari, and G. Andersson. Building control and storage management with dynamic tariffs for shaping demand response. In Proceedings of the IEEE PES Conference on Innovative Smart Grid Technologies (ISGT) Europe, Manchester, UK, Dec. 2011.

[13] J. Joo and M.D. Ilic. Adaptive load management (alm) in electric power systems. In Proceedings of the International Conference on Networking, Sensing and Control, pages 637-642, Chicago, Ilinois, USA, April 2010.

[14] R. Kota, G. Chalkiadakis, V. Robu, A. Rogers, and N. R. Jennings. Cooperatives for demand side management. In Conference on Prestigious Applications of Intelligent Systems (PAIS @ ECAI 2011), pages 969-974, Montpellier, France, August 2012.

[15] S. Ramchurn, P. Vytelingum, A. Rogers, and N. Jennings. Agent-based control for decentralised demand side management in the smart grid. In International Conference on Autonomous Agents and Multiagent Systems (AAMAS 2011), pages 5-12, Taipei, Taiwan, May 2011.

[16] M. Vinyals, F. Bistaffa, A. Farinelli, and A. Rogers. Coalitional energy purchasing in the smart grid. In Proceedings of the IEEE International Energy Conference \& Exhibition (ENERGYCON 2012), 2012.

[17] F. Oldewurtel et al. Energy efficient building climate control using stochastic model predictive control and weather predictions. In Proceedings of the American Control Conference, Baltimore, USA, June 2010.

[18] F. Oldewurtel. Stochastic model predictive control for energy efficient building climate control. PhD thesis, ETH Zürich, Switzerland, 2011.

[19] T. H. Cormen, C. E. Leiserson, R. L. Rivest, and C. Stein. Introduction to Algorithms. The MIT Press, third edition, 2009.

[20] S. Koch, F. S. Barcenas, and G. Andersson. Using controllable thermal household appliances for wind forecast error reduction. In Proceedings of the IFAC Conference on Control Methodologies and Technology for Energy Efficiency, Vilamoura, Portugal, March 2010.

[21] U. Focken et al. Short-term prediction of the aggregated power output of wind farms - a statistical analysis of the reduction of the prediction error by spatial smoothing effects. Journal of Wind Engineering and Industrial Aerodynamics, 90(3):231-246, March 2002.

[22] Meteoswiss. Available online at www.meteoschweiz.ch, last accessed 31.10.2011.

[23] European Power Exchange (EPEX). Available online at www.epexspot.com, last accessed 05.02.2012.

[24] Balance Group Model Introduction. Available online at www.swissgrid.ch, last accessed 05.02.2012. 